\title{
Cardiovascular response patterns during choice reaction time
}

\author{
J. RICHARD JENNINGS \\ University of Pittsburgh, Pittsburgh, Pennsylvania 15260 \\ JOHN SCHROT \\ Navy Medical Research Institute, Bethesda, Maryland 20014
}

and

\author{
CHARLES C. WOOD \\ VA Medical Center, West Haven, Connecticut 06516
}

\begin{abstract}
Vascular responses during a paced choice reaction time task (speed accuracy tradeoff) were compared to concomitant heart-rate responses. In this type of task, cardiac deceleration prior to the discriminative stimulus is known to be under vagal control. Examination of sympathetically controlled vascular responses asked whether simultaneous vagal and sympathetic responses could be observed within the cardiovascular system. Eighteen human volunteers performed the speed-accuracy tradeoff task under one of two monetary payoff conditions. As in previous work, cardiac deceleration was greater under conditions of greater speed emphasis. Parallel effects were observed in the signal amplitude of a photometric index of cutaneous flow. Between sessions with constant monetary payoff, average heart rate decreased and the time between peripheral pulse signals (pulse transit time, PTT) increased. The latter effect was not present when the monetary payoff was altered for the second session. The results suggest that the intensity of second-by-second vagal heart-rate responses are directly related to the intensity of sympathetic inhibition of cutaneous blood flow. Longer term adjustments in sympathetic outflow may be reflected in the between-session PTT results.
\end{abstract}

The subjective experience of heightened alertness and preparedness is frequently reported just prior to the signal to respond in a reaction time task. This experience is not, however, associated with a preparatory speeding of heart rate but, rather, with a welldefined cardiac deceleration (see reviews in Elliott, 1972; Hahn, 1973; B. C. Lacey \& J. I. Lacey, 1974; J. I. Lacey \& B. C. Lacey, 1974). The heart-rate slowing seems to be of vagal origin (Obrist, Webb, Sutterer, \& Howard, 1970). We (Jennings \& Wood, 1977) have shown that the size and timing of these vagal responses are altered when the speed and accuracy of choice responses are varied. In particular, trials requiring faster responses are associated with relatively greater cardiac deceleration than those requiring relatively slower responses. Furthermore, volunteers report that relatively faster responses require more effort. A speeded, effortful task would be expected by many (e.g., Kahneman, 1973), however,

Requests for reprints should be sent to J. Richard Jennings, who is at the Department of Psychiatry, University of Pittsburgh, Westion Psychiatric Institute and Clinic, 3811 O'Hara Street, Pittsburgh, Pennsylvania 15261. The work described was supported and data collected at the Walter Reed Army Institute of Research, Washington, D.C. to be associated with significant sympathetic, as opposed to vagal, activation. Is it possible to observe concomitant sympathetic and parasympathetic activation under such conditions? Indeed, the Laceys (J. I. Lacey, Kagan, B. C. Lacey, \& Moss, 1963) noted a number of years ago that cardiac deceleration could be observed associated with a sympathetic skin conductance response. The current experiment asks whether variations in the vagal cardiac deceleration response are associated with vascular changes suggesting sympathetic activation.

A number of investigators have been interested in the question of whether the vagal heart-rate response is accompanied by sympathetic effects on the remainder of the cardiovascular system. Using the venous occlusion technique, Williams, Bittker, Buschbaum, and Wynne (1975) reported a heart-rate deceleration accompanied by vascular changes indicative of sympathetic excitation: reduced forearm blood flow and digital pulse volume. Despite use of similar sensory input tasks, however, this finding has not always been replicated (Cohen \& Johnson, 1977; Williams, Poon, \& Burdette, 1977). A potential problem with these studies is the temporal averaging of vascular events required by the venous occlusion technique. A phasic cardiac deceleration lasting a few 
seconds is being related to a blood-flow reading which is necessarily averaged over a 10 - to $15-\mathrm{sec}$ period. Obrist, Lawler, Howard, Smithson, Martin, and Manning (1974) were able to assess beat-by-beat events, but peripheral vascular events were not directly measured. Invasive blood pressure and an indirect index of carotid $\mathrm{dp} / \mathrm{dt}$ were collected in conjunction with heart rate. The responses were observed during the foreperiod of a stressful reaction time task similar to that employed in the current experiment. Significant increases in blood pressure were associated with, but not contingent upon, the cardiac deceleration occurring during the foreperiod. The interpretation of Obrist et al. (1974) suggested a vagally induced decrease in heart rate accompanied by a sympathetically induced increase in peripheral vascular resistance. These results were obtained, however, in a sample selected for cardiovascular reactivity. The possible limitation on generalization is suggested by at least one experiment in animals showing decreases in blood pressure accompanying anticipatory heart-rate deceleration (Powell \& Kazis, 1976).

The current experiment examined sympathetic and vagal cardiovascular responses using a task similar to that of Obrist et al. (1974) but using peripheral vascular, rather than invasive blood-pressure, measures. A newly developed photometric technique (Jennings, Tahmoush, \& Redmond, in press) was used to index cutaneous blood flow and pulse-wave velocity. These parameters are influenced by sympathetic nervous system activity in contrast to heart rate, which is influenced by both sympathetic and parasympathetic activity. Transient changes in blood flow are primarily a function of vessel diameter and pulse pressure. Thus, both cardiac (primarily beta adrenergic) and vascular (primarily alpha adrenergic) sympathetic control will alter blood flow. At a constant pressure, pulse-wave velocity is a function of vessel radius, wall thickness, and an elasticity coefficient. Modification of all these parameters, but primarily vessel radius, occurs with sympathetic activity (Aars, 1971; Dobrin \& Rovrick, 1969; Farrar, Green, Bond, Wagner, \& Gobbee, 1978; Lyon \& Sands, 1925).

The noninvasive measurement of beat-by-beat blood flow is difficult, and no one technique can presently claim to provide accurate measures (see Hwang \& Norman, 1977). We have worked with a photometric device similar to devices previously termed photoplethysmographs. In fact, however, our in vitro studies have shown that photo devices are relatively insensitive to blood volume, but quite sensitive to pulsatile blood flow. Thus, in a rigid glass system pulsatile flow registered by an electromagnetic flowmeter and the photometric device were found to be highly correlated (Jennings et al., in press). Although the photometric device has not been shown to measure only flow, an interpretation of its output as related to flow seems reasonable at the current time. Thus, changes in photometric pulse amplitude will be interpreted in terms of sympathetic influences on blood flow.

An index of pulse-wave velocity may be obtained through the use of two photometric devices. The basic measure is the time between pulse signals obtained from devices at different arterial locations. With the distance along the artery known, this time may be converted to pulse-wave velocity. In the current experiment, this distance, although approximately equal between groups of volunteers, is unknown. As a result, only the time between signals will be employed as the dependent measure. A related time, that between the $R$ wave of the electrocardiogram and a peripheral vascular pulse, pulse propagation time has been used by psychophysiologists as a measure of blood pressure. Obrist, Light, McCubbin, Hutcheson, and Hoffer (1979) and Steptoe, Smulyan, and Gribbin (1976), for example, have shown high correlations between pulse transit times and either systolic or mean arterial pressure. Our approach has been oriented to obtaining a measure of peripheral vascular activity rather than a measure of blood pressure. With this orientation, the time between peripheral pulses at different arterial locations is a more relevant measure than $R$ wave to pulse times.

The experimental task was designed, first, to elicit task-induced heart rate deceleration of different magnitudes and relate these to vascular changes and, second, to examine the influence of a subsequent manipulation increasing the monetary rewards for the desired performance. Such an increase in monetary reward is typically viewed as an incentive leading to greater task involvement (e.g., Manuck \& Garland, 1979; Manuck, Harvey, Lechleiter, \& Neal, 1978). If sympathetic activity is assumed to vary with task involvement, the current experiment examines varying degrees of sympathetic influences on the cardiovascular system in the context of the task-induced heartrate deceleration.

\section{METHOD}

\section{Volunteers}

Eighteen young (mean age $=24$ ) adults ( 11 females and 7 males) were paid volunteers. Volunteers were assigned randomly to one of four groups defined by the incentive and target-width conditions defined below.

\section{Procedure and task}

A speed-accuracy tradeoff paradigm was used. Wood and Jennings (1976) provide a full description of this paradigm and the rationale for its use. A choice reaction time task is performed with payoffs for varying response speeds. A 1,000 - or $1,100-\mathrm{Hz}$ tone (at $78 \mathrm{dbA}$ ) signaled the lift of a digit of the right or left hand from a response key. A right-hand response to the $1,100-\mathrm{Hz}$ tone and a left-hand response to the $1,000-\mathrm{Hz}$ tone were correct. Tones were terminated by the response. For a block of 64 trials, volunteers were paid for correct responses within a given time range (e.g., 
125 to $175 \mathrm{msec}$ ). These time ranges were termed RT targets and were defined by the midpoints of the range at 150, 200, 250, 300, and $350 \mathrm{msec}$. All volunteers were well trained in the speedaccuracy task. Prior to the experimental session, volunteers had performed the speed-accuracy task during 1-h sessions on 5 separate days. In each session, volunteers received a block of 64 trials at each of the RT targets. The order of the target blocks was randomized. For one-half of the volunteers, the target range was the target $\pm 25 \mathrm{msec}$; for the other half, the target $\pm 50 \mathrm{msec}$. This target-width variable had no clear effects on performance or physiological responses and will not be discussed further. Speed and correctness feedback followed $200 \mathrm{msec}$ after each response. Trials (tone stimuli) were initiated at the R-wave of the electrocardiogram following a 7 -sec intertrial interval.

The experimental day required two sessions, and began with a separate warm-up block using a $250-\mathrm{msec}$ target. All volunteers received the same payoff, $2 \mathrm{c}$ for correct responses in the target range, during the first session. A break followed, during which the instructions for the second session were given. For the lowincentive group $(n=9)$, the second session was identical to the first. For the high-incentive group $(n=9)$, the payoffs were altered: $5 \mathrm{c}$ was received for each correct response in the target range, but $2 \mathrm{c}$ was lost for all other responses. The high-incentive payoff was introduced as "an opportunity to make substantially more money, but at the risk of losing money." During both sessions, monetary gains or losses were announced to the volunteer at the end of each block of trials. Following the second session, volunteers were interviewed concerning their degree of involvement in the task and the relative effort required by the different target conditions.

Electrocardiogram (EKG) electrodes were attached to the sternum and left lateral margin of the chest (Lexington disposable electrodes and paste) for all sessions. Photometric devices for peripheral vasculature measurement were attached on the distal surface of the thumb of the right hand and over the left carotid artery below the jaw. Attachment and a device description is available in Tahmoush, Jennings, Lee, Camp, and Weber (1976). In addition, a thermistor, cemented into an earring, was placed within the nasal cavity to monitor breathing.

\section{Data Reduction}

All physiological data were recorded on analog tape and reduced using computer techniques. The R-wave of the electrocardiogram was detected with a laboratory-built peak detector and the R-R or interbeat-interval (IBI) digitized. Analyses were performed on the IBIs for the three beats preceding the stimulus, the beat of stimulus occurrence, and the three beats following the stimulus. Considering the first beat, analyzed as Beat 1 , the stimulus occurred on the $R$-wave ending Beat 4 and beginning Beat 5 . Photometric signals occurring during Beat 5 were averaged by time-locking on the $\mathrm{R}$-wave initiating the tone stimulus. The analog signal for each trial was sampled once every $1.9 \mathrm{msec} ; 64$ samples were taken. These samples were averaged across the 64 trials of a block. The averages were then scored for the time of onset of systole and peak amplitude. Due to the time-locking employed, onset of systole also defined time from R-wave. A pulse-wave-velocity-related measure was derived by subtracting carotid onset time from thumb onset time. This measure, termed pulse transit time (PTT), eliminates intracardiac delay time (which must be identical for both pulses). A pulse-wave velocity from a single arterial tree would be preferable, but the carotid site gave better recording than sites on the radial and brachial arteries. Respiration was monitored to insure that volunteers were breathing normally. Analog tape-recorder limitation did not allow the recording of this variable. Jennings and Wood (1977) report respiratory effects found in the speedaccuracy tradeoff paradigm.

\section{RESULTS}

\section{Performance and Incentive Pay}

The clear control over RT and accuracy resulting
Table 1

Performance as a Function of Target, Session, and Incentive Group

\begin{tabular}{|c|c|c|c|c|c|}
\hline & \multicolumn{5}{|c|}{ Targets (in Milliseconds) } \\
\hline & 150 & 200 & 250 & 300 & 350 \\
\hline & \multicolumn{5}{|c|}{ Low Incentive: Session 1} \\
\hline \multirow[t]{2}{*}{$\begin{array}{l}\mathrm{RT} \\
\mathrm{PC}\end{array}$} & $\begin{array}{r}211 \\
53\end{array}$ & $\begin{array}{r}237 \\
65\end{array}$ & $\begin{array}{r}277 \\
74\end{array}$ & $\begin{array}{r}309 \\
85\end{array}$ & $\begin{array}{r}352 \\
90\end{array}$ \\
\hline & \multicolumn{5}{|c|}{ Low Incentive: Session 2} \\
\hline \multirow[t]{2}{*}{ PC } & $\begin{array}{r}227 \\
55\end{array}$ & $\begin{array}{r}251 \\
65\end{array}$ & $\begin{array}{r}275 \\
74\end{array}$ & $\begin{array}{r}304 \\
83\end{array}$ & $\begin{array}{r}340 \\
84\end{array}$ \\
\hline & \multicolumn{5}{|c|}{ High Incentive: Session 1} \\
\hline \multirow[t]{2}{*}{ PC } & $\begin{array}{r}230 \\
59\end{array}$ & $\begin{array}{r}253 \\
63\end{array}$ & $\begin{array}{r}289 \\
69\end{array}$ & $\begin{array}{r}315 \\
79\end{array}$ & $\begin{array}{r}358 \\
87\end{array}$ \\
\hline & \multicolumn{5}{|c|}{ High Incentive: Session 2} \\
\hline $\begin{array}{l}\text { RT } \\
\text { PC }\end{array}$ & $\begin{array}{r}224 \\
57 \\
\end{array}$ & $\begin{array}{r}254 \\
61 \\
\end{array}$ & $\begin{array}{r}291 \\
76\end{array}$ & $\begin{array}{r}310 \\
75\end{array}$ & $\begin{array}{r}354 \\
88\end{array}$ \\
\hline
\end{tabular}

Note-Reaction time (RT) is expressed in milliseconds; accuracy of proportion correct (PC) is the number of correct choice responses divided by the total number of trials.

from the target manipulation is shown in Table 1. Increases in RT and accuracy were linear with increasing RT target. As described in previous publications (Jennings \& Wood, 1977; Wood \& Jennings, 1976), speed-accuracy tradeoff functions were formed by linear fits of accuracy data points plotted as a function of mean RT for different targets. The average $\mathbf{R}^{2}$ goodness-of-fit index was .79.

As suggested in Table 1, the change in incentive between sessions had no striking effect on performance. This impression and the effects of RT target were verified in analyses of variance on RT and accuracy scores which had factors for Target, Incentive, and Session. A rejection region of $p<.05$ was used throughout all analyses. Due to potential inhomogeneity of variance, the degrees of freedom for all repeated measures factors were corrected using the Greenhouse-Geisser procedure (Jennings \& Wood, 1976). In the RT analysis, the only factor significant was Target $[F(\operatorname{adj} .1,17)=89.81]$. Similarly, only the Target factor was significant in the accuracy analysis $[F(\operatorname{adj} .1,17)=50.29]$. An analysis of speed-accuracy tradeoff function parameters failed to show any significant effect of session or incentive.

Postexperimental ratings also showed clear target effects and only suggestive incentive effects. As might be expected on the dimension of effort, targets were rated in descending order, with the 150 target rated as requiring the most effort (Friedman $\chi^{2}=40.7$, $\mathrm{df}=4$ ). The mean rating of task involvement was slightly higher in high-incentive volunteers (6.8 vs. 6.5 ), but not significantly so. General involvement in the experimental task was rated from a 0 of no involvement to a 10 of highest involvement previously experienced. Unfortunately, ratings of Session 2 relative to Session 1 were not obtained. 


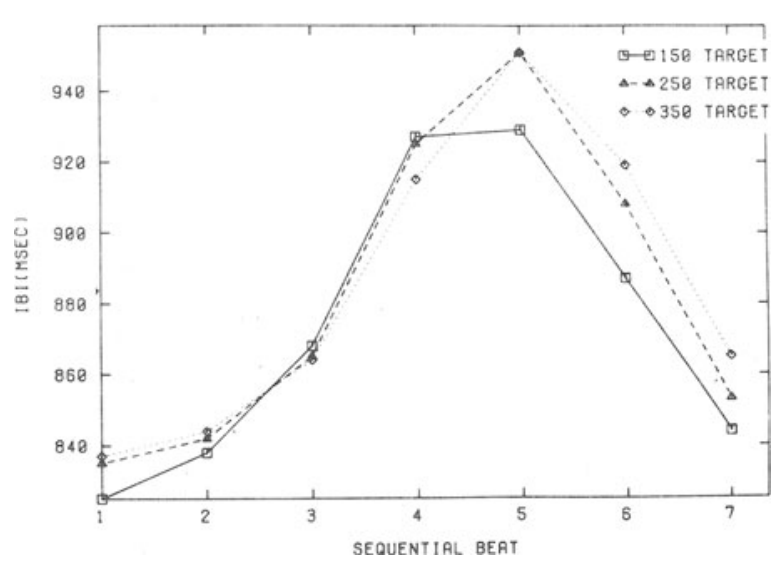

Figure 1. Mean interbeat interval values for successive heart beats before, during, and after $\mathrm{RT}$ performance. The auditory choice stimulus occurred on the $R$-wave of the heart beat ending Beat 4; that is, at number 4 on the abscissa. Results are presented separately for blocks of 64 trials at RT targets of 150, 250, and 350 msec.

In the high-incentive group, the payoff manipulation altered the range of earnings but not average earnings. For Session 1, low- and high-incentive groups earned approximately the same amount, $\$ 2.74$ and $\$ 2.60$, respectively. Similar means were found for Session 2, $\$ 2.63$ and $\$ 2.19$; however, the range of earnings for the low-incentive group was $\$ 1.62$ to $\$ 3.76$ and $-\$ .68$ to $\$ 6.51$ for the high-incentive group.

\section{Physiological Variables}

Target effects and the IBI response over beats. The heart-rate response during the task was the expected deceleration followed by accelerative recovery. Figure 1 presents the mean beat-by-beat heart-rate response (averaged across incentive) for three target conditions $(150,250$, and $350 \mathrm{msec})$. Heart rate is expressed as IBI, reciprocal of heart rate, for example, in Figure 1 an increase in IBI indicates a deceleration of heart rate. The tone stimulus occurs at the end of Beat 4. The expected cardiac deceleration occurs up to the beat in which the stimulus is presented. Accelerative recovery follows the response.

The effect of the target, session, and incentive var- iables will be evaluated by examining the effects on both cardiac and vascular variables. Table 2 presents the effect of the target variable. The values associated with the heart beat of stimulus occurrence (Beat 4) are tabulated. Vascular measures were analyzed solely at this beat. The major effects shown in the table are for thumb-pulse amplitude and heart-rate deceleration. As progressively slower target values are examined (150 toward $350 \mathrm{msec}$ ), thumb-pulse amplitude and degree of deceleration decrease. The pulseamplitude effect seems to be localized to the thumb. Carotid-pulse changes are small and irregular, and pulse transit time changes are essentially nonexistent.

The differences apparent in Table 2 were evaluated for statistical significance using analysis of variance. For IBI, a four-way analysis of variance, with Incentive Group, Session, Target, and Beat, was used. The latter three factors were within-subject and the epsilon correction for inhomogeneity of covariance was applied as appropriate. As applied to IBI, factors were significant $(p<.05)$ for Session $(F=9.44$, $\mathrm{df}=1 / 16)$, Beats $(\mathrm{F}=53.74$, adj. $\mathrm{df}=1 / 16)$, and Target by Beats $(F=4.78$, adj. $\mathrm{df}=1 / 16)$. The target effect, which is the result relevant to Table 2, reflects not only the decelerative differences in Table 2, but also differences in the accelerative recovery limb (see Figure 1). The results replicate those of Jennings and Wood (1977) by showing that relatively faster targets are associated with (a) greater accelerative recovery and (b) a shortened latency of accelerative recovery. (See Jennings \& Wood, 1977, for a discussion of point $b$ in terms of cardiac cycle effects.) For our current focus on the beat of stimulus occurrence, however, the decelerative difference shown in Table 2 should be directly tested. Therefore, an analysis of Target, Session, and Incentive effects on the Beat 4Beat 1 difference score was performed which showed the expected significant effect of Target $(F=6.83$, adj. $\mathrm{df}=1 / 17$ ).

Although photometric pulse amplitudes were not calibrated in this study, the relative value of amplitudes for within-subject comparisons are meaningful. The target variable is such a variable, and the target effect shown in Table 2 was shown to be statistically significant for thumb-pulse but not carotidpulse amplitudes. Amplitudes were scaled in arbitrary units which were submitted to a square-root

Table 2

Effects of Target on Cardiovascular Variables Measured at the Heart Beat Initiating the RT Stimulus

\begin{tabular}{lrrrrr}
\hline & \multicolumn{5}{c}{ Target Values (in Milliseconds) } \\
\cline { 2 - 5 } & 150 & 200 & 250 & 300 & 350 \\
\hline Pulse Transit Time (in Milliseconds) & 46.3 & 47.5 & 51.8 & 58.0 & 53.1 \\
Thumb-Pulse Amplitude (Arbitrary Units) & 100 & 92 & 92 & 74 & $74^{*}$ \\
Carotid-Pulse Amplitude (Arbitrary Units) & 96 & 100 & 92 & 100 & 90 \\
Deceleration at Stimulus (Beat 4-Beat 1) & 102 & 101 & 91 & 84 & $78^{*}$ \\
\hline
\end{tabular}

*Statistically significant difference (see text). 
transformation in order to normalize the distributions. A repeated measures analysis of variance with factors for Incentive, Session, and Target showed a significant Target effect on thumb-pulse amplitude $(F=5.15$, adj. $d f=1 / 17)$. Target effects were not present in analyses of $\mathbf{R}$-wave to carotid- or thumbpulse onset time intervals.

Incentive and session effects. The second session examined the question of whether cardiovascular reactivity was altered by a change in monetary payoff. As suggested by Table 3 , session and incentive had overall effects that did not interact with the target variable. A large increase in PTT occurred in the lowbut not in the high-incentive groups (Incentive by Session, $F=7.65$, adj. $d f=1 / 17)$. Values of the other cardiovascular indices in Table 3 remained relatively stable, although a suggestion of greater deceleration relative to Session 1 is indicated in the high-incentive group. The previously presented analysis of IBI as well as separate analyses on Session 2 data failed, however, to support this trend. The analyses did clearly support the significance of an increase in average IBI from Session 1, $867 \mathrm{msec}$ to Session 2, $894 \mathrm{msec}$.

A second perspective on the incentive-session effect may be gained by examining joint changes in physiological variables. Presumably, changes induced by a single variable, such as fatigue, might be reflected by correlated changes in physiological measures. Such correlation could be disrupted by the addition of another variable, such as incentive. Spearman rank order correlations computed between degree of deceleration and PTT showed such a pattern of results. Decreases in PTT between sessions were significantly correlated $(\mathrm{rho}=.72)$ with increased cardiac deceleration in the low- but not the high-incentive group (rho $=-.17)$. Correlation among the remaining cardiovascular variables failed to approach statistical significance.

\section{DISCUSSION}

The primary aim of this experiment was to demonstrate a patterning in autonomically controlled cardiovascular responses. In particular, evidence was sought showing sympathetic-like changes in vascular variables during the occurrence of a vagally induced heart-rate deceleration. Over the course of the experiment, such joint changes in sympathetic and vagal influences were observed; however, vagal excitatory and sympathetic inhibitory influences were predominant in phasic or second-to-second responses to the task. Sympathetic influences, on the other hand, were primarily tonic, that is, lasting a number of minutes. For purposes of discussion, responses attributed primarily to vagal excitation or sympathetic inhibition will be separated from responses attributed to sympathetic excitation or vagal inhibition.
Table 3

Cardiovascular Parameters in Low- and High-Incentive Groups from Session Pre- (Session 1) and Postincentive (Session 2) Instructions

\begin{tabular}{lrrrrc}
\hline & \multicolumn{3}{c}{ Incentive } \\
\cline { 2 - 3 } & Preincentive & Postincentive \\
\cline { 2 - 3 } \cline { 5 - 6 } & Low & High & & Low & High \\
\hline Pulse Transit Time (in Milliseconds) & 49 & 39 & 39 & $74^{*}$ \\
Thumb-Pulse Amplitude & 72 & 127 & 117 & $65^{* *}$ \\
Carotid-Pulse Amplitude & 100 & 98 & 99 & $108 * *$ \\
Deceleration (IBI 4-IBI 1) & 93 & 83 & 94 & 95 \\
\hline
\end{tabular}

*Session by Incentive interaction significant. **Betweengroups comparisons are impossible without calibration; withinsubject session effect is not significant.

\section{Vagal Influences}

Vagal influences appeared to be responsible for the phasic heart-rate deceleration induced by the RT task and the variations in depth of deceleration induced by the RT targets. The vagal origin of the phasic deceleration has been most clearly demonstrated by Obrist (e.g., Obrist et al., 1974). In the current experiment, depth of deceleration was altered by RT target such that targets requiring relatively faster RTs were associated with relatively greater prestimulus deceleration. These differences suggest vagal excitation as they are the opposite of that expected from sympathetic activation. Jennings and Wood (1977) have previously suggested that the time course of vagal excitation may be closely related to the change in heart-rate response with RT target.

Vascular effects seemed to reflect a sympathetic inhibition concomitant with the vagal excitation of the heart. As vascular results were available only for the beat of stimulus occurrence, a comparison of phasic deceleration with a phasic vascular response is not possible. Larger pulse amplitudes were, however, associated with targets requiring faster responses. Interpreting these amplitudes as related to flow, and thus to vessel diameter, suggests that sympathetic inhibition was relatively stronger for the fast targets. An alternative interpretation might suggest that pulse pressure was higher for fast targets in the presence of a constant cutaneous vascular resistance. This alternative interpretation is questioned to the extent that $\mathbf{R}$-wave to carotid-pulse times reflect systolic blood pressure (Obrist et al., 1979). The R-wave to carotid times for the current task were essentially constant across target conditions.

As noted earlier, most previous work has suggested that a decrease in forearm blood flow may occur in "sensory input" tasks (Cohen \& Johnson, 1977; Williams et al., 1975, 1977; Williams, Bauknight, Cleveland, \& Jackson, Note 1). Forearm blood flow reflects primarily blood flow to the muscles, while blood flow to the distal portion of the thumb is pri- 
marily cutaneous blood flow. Previous and current work is consistent if a regional distribution of blood during anticipation is suggested with vasoconstriction in the muscle and dilation in the skin. The digital pulse volume results of Williams et al. (1975) are an as yet unexplained exception to this generalization.

From a psychophysiological perspective, a state of heightened preparedness in anticipation of a task appears to be related to vagal excitation. Relative to simple concepts of a sympathetic "arousing" nervous system antagonized by a parasympathetic "relaxing" nervous system, the active nature of this concomitant of vagal activity is of interest. We have previously suggested that the state of preparedness may be interpreted as holding central processing capacity available (Jennings, Lawrence, \& Kasper, 1978).

\section{Sympathetic Influences}

Evidence for sympathetic influences on the cardiovascular system was seen in the interaction of session and incentive. In the absence of any change in incentive, that is, in the low-incentive group, a reduction in sympathetic activity in the second session was indicated by decreases in mean heart rate and increases in PTT. Although mean phasic cardiac deceleration was essentially unchanged between sessions, individual variation in the degree of Session 2 deceleration was significantly correlated to the change in PTT between sessions. This suggests a decrease in sympathetic activity that influenced both the heart and vasculature. In contrast, when incentive increased between sessions, that is, in the high-incentive group, PTT remained the same rather than increasing. In agreement with the results of J. I. Lacey (1972) and Lawler, Obrist, and Lawler (1976), a trend toward greater phasic deceleration also occurred with heightened incentive. As might be expected, if the deceleration was due to lengthened or maintained vagal activity, phasic deceleration in the high-incentive group was not correlated to between session changes in PTT. Incentive served to maintain vascular sympathetic activity in the context of a maintained or heightened phasic vagal effect on heart rate.

Obrist and his colleagues (Obrist, Gaebelein, Teller, Langer, Gringolo, Light, \& McCubbin, 1978; Obrist et al., 1974; Obrist, Light, McCubbin, Hutcheson, \& Hoffer, 1979) have, for the last few years, been examining stress-induced sympathetic influences on the heart. One situation has been a reaction time task with electric shock contingent upon performance. This task has consistently produced increases in average heart rate, systolic blood pressure, and rate of change of pressure over the carotid artery. As in the current task, however, second-by-second myocardial effects appeared primarily due to vagal, rather than sympathetic, influence. Relatively tonic sympathetic myocardial effects seem to occur, but even in this time frame vascular sympathetic effects, as indicated by mean blood pressure and PTT, are more pronounced. Thus, the interpretation of the current results is generally in accord with the results of Obrist and his colleagues.

\section{Cardiovascular Response Patterning}

The current results suggest that potent vagal effects on the cardiovascular system can occur in the context of tonic sympathetic effects. This result is not surprising in the context of the previous reports of specific patterns of autonomic response in human psychophysiology or growing evidence of the extent and precision of neural control of the cardiovascular system as obtained in animal preparations (e.g., Caleresu, Faiers, \& Mogensen, 1975). Sympathetic and parasympathetic nervous control cannot be conceptualized as the inverse of one another. Rather, relative independence must be recognized and differences in initiating conditions, latency, amplitude, and regional specificity of their effects must be described. For the human psychophysiologist, a major hindrance to this effort is the continuing need to first separate sympathetic and parasympathetic effects using noninvasive monitoring techniques. The significance of continuing this effort may be practical as well as theoretical. Certain authors (e.g., Skinner, 1979; Williams, 1978) have suggested a link between cardiac disease and the autonomic changes related to the regulation of sensory input. Although this represents an exciting direction for further exploration, a more modest goal is simply the accurate description of the hemodynamic significance of neural adjustments of the cardiovascular system.

\section{REFERENCE NOTE}

1. Williams, R. B., Jr., Bauknight, T., Cleveland, W., \& Jackson, M. Phasic forearm blood flow responses during the preparatory interval of a reaction time task. Paper presented at the annual meeting of the Society for Psychophysiological Research, San Diego, October 1976.

\section{REFERENCES}

Aars, H. Effect of altered smooth muscle tone on aortic diameter and aortic baroreceptor activity in anaesthetized rabbits. Circulation Research, 1971, 28, 254-262.

Calaresu, F. R., Faiers, A. A., \& Mogensen, G. J. Central neural regulation of heart and blood vessels in mammals. Progress in Neurobiology, 1975, 5, 1-35.

Cohen, D. J., \& Johnson, W. T. Cardiovascular correlates of attention in normal and psychiatrically disturbed children. Archives of General Psychiatry, 1977, 34, 561-564.

Dobrin, P. B., \& Rovrick, A. A. Influence of vascular smooth muscle on contractile mechanics and elasticity of arteries. American Journal of Physiology, 1969, 217, 1644-1651.

ELLIOTT, R. The significance of heart rate for behavior: A critique of Lacey's hypothesis. Journal of Personality and Social Psychology, 1972, 22, 398-409. 
Farrar, D. J., Green, H. D., Bond, M. G., Wagner, W. D. \& Gobbez, R. A. Aortic pulse wave velocity, elasticity, and composition in a nonhuman primate model of atherosclerosis. Circulation Research, 1978, 43, 52-62.

Hahn, W. W. Attention and heart rate: A critical appraisal of the hypothesis of Lacey and Lacey. Psychological Bulletin, $1973,79,59-70$.

Hwang, N. H. C., \& Norman, N. A. Cardiovascular flowdynamics and measurements. Baltimore, Md: University Park Press, 1977.

Jennings, J. R., Lawrence, B. E., \& Kasper, P. Changes in alertness and processing capacity in a serial learning task. Memory \& Cognition, 1978, 6, 45-53.

Jennings, J. R., Tahmoush, A. J., \& Redmond, D. Peripheral vascular measurement. In P. H. Venables \& I. Martin (Eds.), Techniques in psychophysiology. London: Wiley, in press.

Jennings, J. R., \& Wood, C. C. The $\varepsilon$-adjustment procedure for repeated-measures analyses of variance. Psychophysiology, 1976, 13, 277-278.

Jennings, J. R., \& Wood, C. C. Cardiac cycle time effects on performance, phasic cardiac responses, and their intercorrelation in choice reaction time. Psychophysiology, 1977, 14, 297-307.

Kahneman, D. Attention and effort. Englewood Cliffs, N.J: Prentice-Hall, 1973.

LACEY, B. C., \& Lacey, J. I. Studies of heart rate and other bodily processes in sensorimotor behavior. In P. A. Obrist, A. Black, J. Brenner, \& L. DiCara (Eds), Cardiovascular psychophysiology-Current issues in response mechanisms, biofeedback and methodology. Chicago: Aldine-Atherton, 1974.

LACEY, J. I. Some cardiovascular correlates of sensorimotor behavior: Example of visceral afferent feedback? In C. $\mathrm{H}$. Hockman (Ed.), Limbic system mechanisms and autonomic function. Springfield, Ill: Thomas, 1972.

Lacey, J. I., Kagan, J. R., Lacey, B. C., \& Moss, H. A. The visceral level: Situational determinants and behavioral correlates of autonomic response patterns. In P. H. Knapp (Ed.), Expression of the emotion in man. New York: International University Press, 1963.

LACEY, J. I., \& LACEY, B. C. On heart rate responses and behavior: A reply to Elliott. Journal of Personality and Social Psychology, 1974, 30, 1-18.

Lawler, K. A., Obrist, P. A., \& Lawler, J. E. Cardiac and somatic response patterns during a reaction time task in children and adults. Psychophysiology, 1976, 13, 448-455.

Lyon, D. M., \& SANDS, J. Studies in pulse wave velocity: IV. Effect of adrenalin on pulse wave velocity. American Journal of Physiology, 1925, 71, 534-542.

Manuck, S. B., \& Garland, F. N. Coronary prone behavior pattern, task incentive, and cardiovascular response. Psychophysiology, 1979, 16, 136-142.

Manuck, S. B., Harvey, A. H., Lechleiter, S. L., \& Neal,
K. S. Effects of coping on blood pressure responses to threat of aversive stimulation. Psychophysiology, 1978, 15, 544-549.

Obrist, P. A., Gaebelein, C. J., Teller, E. S., Langer, A. W. Gringolo, A., Light, K. C., \& McCubbin, J. A. The relationship among heart rate carotid $\mathrm{dp} / \mathrm{dt}$ and blood pressure in humans as a function of the type of stress. Psychophysiology, $1978,15,102-115$.

Obrist, P. A., Lawler, J. E., Howard, J. L., Smithson, K. W., Martin, P. L., \& Manning, J. Sympathetic influences on cardiac rate and contractility during acute stress in humans. Psychophysiology, 1974, 11, 405-427.

Obrist, P. A., Light, K. C., McCubbin, J. A., Hutcheson, J. S., \& Hoffer, J. L. Pulse transit time: Relationship to blood pressure and myocardial performance. Psychophysiology, 1979, 16, 292-301.

Obrist, P. A., Webb, R. A., Sutterer, J. R., \& Howard, J. L. Cardiac deceleration and reaction time: An evaluation of two hypotheses. Psychophysiology, 1970, 6, 695-706.

Powell, D. A., \& Kazis, E. Blood pressure and heart rate changes accompanying classical eyeblink conditioning in the rabbit (Oryctolagus cuniculus). Psychophysiology, 1976, 13, 441-448.

Skinner, J. E. The role of the frontal cortex in the regulation of cardiac vulnerability to ventricular fibrillation: A new concept of Cannon's cerebral defense mechanism. In E. Donchin, G. Galbraith, \& M. Kietzman (Eds.), Neurophysiology and psychology: Basic mechanisms and clinical application. New York: Academic Press, 1979.

Ste Ptoe, A., Smulyan, H., \& Gribiin, B. Pulse wave velocity and blood pressure change: Calibration and applications. Psychophysiology, 1976, 13, 488-493.

Tahmoush, A. J., Jennings, J. R., Lee, A. L., Camp, S., \& WEBER, F. Characteristics of a light emitting diode-transistor photoplethysmograph. Psychophysiology, 1976, 75, 277-278.

Williams, R. B., JR. Psychophysiological processes, the coronary prone behavior pattern, and coronary heart disease. In T. M. Dembroski, S. M. Weiss, J. L. Shields, S. G. Haynes, \& M. Feinleib (Eds.), Coronary-prone behavior. New York: Springer-Verlag, 1978

Williams, R. B., Jr., Bittker, T. E., Buchsbaum, M. S., \& Wynne, L. C. Cardiovascular and neurophysiologic correlates of sensory intake and rejection in effect of cognitive tasks. Psychophysiology, 1975, 12, 427-433.

Williams, R. B., JR., Poon, L. W., \& Burdette, L. J. Locus of control and vasomotor response to sensory processing. Psychosomatic Medicine, 1977, 39, 127-133.

Wood, C. C., \& Jennings, J. R. Speed-accuracy trade off functions in choice reaction time: Experimental designs and computational procedures. Perception \& Psychophysics, 1976, 19, 92-101.

(Received for publication August 28, 1979; revision accepted December 19, 1979.) 